
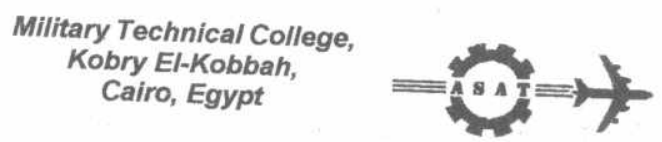
$g^{\text {th }}$ International Conference
On Aerospace Sciences \& Aviation Technology

\title{
DETERMINATION OF REFRACTIVE INDEX AND MATERIAL
DISPERSION OF STEP INDEX OPTICAL DISPERSION OF STEP INDEX OPTICAL FIBERS USING VARIABLE
WAVELENGTH INTERFEROMETRIC WAVELENGTH INTERFEROMETRIC TECHNIQUE
}

Shanshoul M. A. , Gerges A.S. ", Mehanna E. A.

\begin{abstract}
:
The variable wavelength interferometric technique is used to determine the refractive
index and material dispersion of [Ge-B doped $\left.\mathrm{SiO}_{2}\right]$ step index modified Pluta polarizing interference-B doped $\mathrm{SiO}_{2}$ ] step index optical fiber. The curve of a step index optical fiber, which is is ised to measure the dispen mechanisms in optical fibers. Microinterich is important in understanding, the loss
\end{abstract}

\section{KEY WORDS:} Engineering physics, Optics, Step Index Optical Fiber, Optical fiber communication,
Refractive index, Material dispersion.

\footnotetext{
* P.G.S. Syrian Arab Army

** Assoc. Prof. Dr. Egyptian Army Forces.

Prof. Dr. Egyptian Army Forces.
} 


\section{1- INTRODUCTION}

Optical fibers have developed rapidly in the forty years since the concept was first practically demonstrated. They have different applications such as medical endscopy and communication systems. The optical fiber is a two component medium in which the fiber core is used as a transmitting medium and the cladding provides a reflecting surface and protects the core from physical damage. The transmission of light through an optical fiber is due to the fundamental principle of total internal refraction [1]. So, the refractive index of the optical fiber core is the basic parameter that describes the properties of the fiber. It allows us to determine some parameters such as; the fiber numerical aperture, the number of propagation modes within the fiber core and the dispersion properties of the fiber.

Many authors have applied interferometric techniques to determine the optical properties of fibers. Pluta (1972) [2] used his double refracting interference microscope with variable amount and direction of wavefront shear to study the refractive indices and birefringence of synthetic polymer fibers. Hamza and Sikorski (1978)[3] used this microscope to determine the optical anisotropy of Kevlar (poly-pphenylene terephthalamide) fibers. Hamza (1980)[4] extended the applications of the Pluta microscope to measure the mean refractive indices and birefringence of fibers with irregular transverse sections. Zurek and Zakrzewski (1983)[5] determined the refractive indices and birefringence of cotton fibers using the Pluta microscope. Hamza et al. [6-9] Used Pluta polarizing interference microscope for different fibers with regular and irregular transverse sections to determine its refractive indices and birefringence. Hamza et al.[10] (1994) used this microscope to study the refractive index profiles of cylindrical fiber having core-cladding structure .

An important component of delay distortion in fiber optic waveguides is produced by wavelength dispersion of the refractive index. Therefore dispersion is as $(d n / d \lambda)$, where $n$ is the refractive index of the matter and $\lambda$ is wavelength of the light used [11]. In fiber optics communication systems material dispersion means the variation of phase velocities, through the fiber due to many factors, which produces pulse broadening and hence limits the maximum number of pulses a fiber can carry in onesecond [12].

A specific microinterferometric method referred to as variable wavelength interferometry (VAWI) was developed by Pluta [13] to measure the fiber refractive index. An ultra-precise object-adopted version (AVAWI) of this technique was proposed for measuring the spectral dispersion curves of the textile fiber [14]. The (VAWI) method includes several specific procedures such as; (VAWI-1) for studying the fibers that produce high optical path difference and (VAWI-2) for studying that fibers produce small optical path difference $[14,15]$.

In this paper we used the (VAWI) technique to measure the refractive index and deter'mine the spectral dispersion curve of step index optical fiber. 


\section{2- THEORETICAL CONSIDERATIONS}

\section{2-1 Principle of the VAWI Method (Variable Wavelength Microinterferometry)}

Let a transparent object (optical fiber) be placed in the object plane of the light transmitting interferometer, If the refractive index of this object is $n$ and that of its surrounding medium is $n$. Let the thickness of the object is $t$, then the optical path difference $\delta$ is [15]:

$\delta=\left(n^{\prime}-n\right) t=m \lambda$

Where $m$ is an integer number ( $m=1,2,3,4 \ldots$ etc), called interference order and $\lambda$ is the wavelength of the light used. The optical path difference must be varied. Consider the variation of $\lambda$ from the red region to the blue region and the first coincidence in the red region is at $\lambda_{1}$ and the second coincidence at $\lambda_{2}$, where $\lambda_{1} \succ \lambda_{2}$. Figure (1) shows the case of coincidence and anti-coincidence of interference fringe shift of the fiber under study with the interference of the surrounding medium.

The situation may be described, in general, by the following equations [15]:

$\delta_{1}=\left(n_{1}^{\prime}-n_{1}\right) t=m_{1} \lambda_{1}$

$\delta_{2}=\left(n_{2}^{\prime}-n_{2}\right) t=\left(m_{1}+q_{2}\right) \lambda_{2}$

$\delta_{3}=\left(n_{3}^{\prime}-n_{3}\right) t=\left(m_{1}+q_{3}\right) \lambda_{3}$

And so

$\delta_{S}=\left(n_{S}^{\prime}-n_{S}\right) t=\left(m_{1}+q_{S}\right) \lambda_{S}$

Where $q$ is an arbitrary integer number take the values $1,2,3$..And $q_{1}=0$ (first coincidence), and $s=2,3,4, m_{1}$ is an integer number, called initial interference order, From equation ( $1 a)$ and ( $1 b)$ the initial interference order $m_{1}$ can be written as follows:

$m_{1}=q_{2} \frac{\lambda_{2}}{N_{21} \lambda_{1}-\lambda_{2}}$

Where $\quad N_{21}=\frac{n_{2}^{\prime}-n_{2}}{n_{1}^{\prime}-n_{1}}$

And from equations: (1a) and (1c) one can write:

$m_{1}=q_{3} \frac{\lambda_{3}}{N_{31} \lambda_{1}-\lambda_{3}}$ 
Where $\quad N_{31}=\frac{n_{3}^{\prime}-n_{3}}{n_{1}^{\prime}-n_{1}}$

Equations $(1 \mathrm{a}, 1 \mathrm{~d})$ can be written in a general form as:

$m_{1}=q_{S} \frac{\lambda_{s}}{N_{S 1} \lambda_{1}-\lambda_{2}}$

(6)

Where $N_{S 1}=\frac{n_{S}^{\prime}-n_{S}}{n_{1}^{\prime}-n_{1}}$

The parameters $N_{21}, N_{31}$ and $N_{S 1}$ are called factors of similarity of the spectral dispersion of refractive indices of the object and the surrounding medium.

If $n(\lambda)=$ constant and $n^{\prime}(\lambda)=$ constant, $N_{21}=N_{31}=N_{S 1} \approx 1$ and equations (2), (4) and (6) take in general a simpler form:

$m_{1}=q_{s} \frac{\lambda_{s}}{\lambda_{1}-\lambda_{s}}$

Equation (8) can be rewritten in terms of interfringe spacing (b) as:

$m_{1}=q_{s} \frac{b_{S}}{b_{1}-b_{S}}$

Where $b=\lambda / \varepsilon, \varepsilon=2 D \tan \alpha, \alpha$ is the apex angle of the Wollaston prism of the Pluta microscope and $D$ is the birefringence of double refracting crystal of the Wollston prism. Equation (9) is valid also when $n(\lambda) \neq$ constant, and $n^{\prime}(\lambda) \neq$ constant, but $n^{\prime}(\lambda)-n(\lambda)=$ constant.

The calculation of the initial interference order $m_{1}$ depends on $N_{21}, N_{31}$ and $N_{S 1}$ These values may be less or more than unity depending on the dispersion of the surrounding medium. Therefore, the value of the initial interference order $m_{1}$ may be fraction number so we must make a good choosing of the correct value of initial interference order.

A specific interferometric method [14], referred to as ultra-precise object-adapted version (AVAWI) are used to measure the spectral dispersion of birefringence and spectral dispersion of the refractive indices of fibres. In this (AVAWI) there are two ways, one of them based on equation (8) and called [AVAWI $(\lambda)$ ] and the other based on equation (9) called [AVAWI (b)] which is our interest. Equation (9) can be written as [14]:

$\left(m_{1}+q_{S}\right) b_{S}=m_{1} b_{1}$ 
Averaging of the product $m b$ that defined as:

$$
\overline{m_{s} b_{s}}=\frac{\sum_{1}^{S}\left(m_{1}+q_{S}\right) b_{S}}{S}=C
$$

Where $S$ is the number of coincidence. Thus the path difference can be determined from the relation:

$$
\delta=\overline{m_{s} b_{s}} \frac{\lambda}{b}=C \frac{\lambda}{b}
$$

Where $\lambda$ is an arbitrary light wavelength and $b$ is the interfringe spacing that corresponding to the used wavelength. In [AVAWI (b)], if $b$ is measured and $\lambda$ is determined, the parameter $C$ can be calculated from equation (11) which is used to obtain several values at any wavelength $\lambda(400 \mathrm{~nm}-700 \mathrm{~nm})$ for the optical path difference from the recurrence relation [14]:

$$
\delta_{s}=C \frac{\lambda_{s}}{b_{s}}
$$

\section{3-EXPERIMENTAL RESULTS AND DISCUSSION}

Pluta $[13,15]$ presented an interferometric method used to investigate fiber known as (VAWI), in which the Pluta microscope is modified by adding a halogen lamp (12V/100W) and interference filter (See figure 2) [16]. To obtain the coincidence of the fringe shift of the fiber with the air fringe, the system is prepared by adjusted the refracting prism in the crossed position with the main Wollaston prism and the subcondenser slit is adjusted to be at right angle to the direction of the wavefront shear.

The fiber is put on the object plane of the microscope, then the interference image of the fiber is splitted into two images of refractive indices $n^{1 !}$ and $n^{\perp}$. The wavelength of the light used is varied via the movement of the wedge interference filter in one direction, the coincidence and anti-coincidence positions of the fringe shift with the air fringes are obtained.

The Double-refracting interference microscope were used to determine spectral dispersion curve for multi-mode step index optical fiber [Ge-B doped $\mathrm{SiO}_{2}$ ]. Figure $(3 . a, b)$ is a microinterferogram of the used fiber, a) gives the case of coincidence position and b) shows the case of anti- coincidence. (VAWI) technique depends on given certain wavelength, calculated from standard calibration graph [16], to obtain coincident and anti-coincident positions of fringes as shown in figure $(3, a, b)$, then using VAWI (b) method to determine the dispersion curve. Table (1) shows the experimental measurement of the refractive index for studied fiber, which calculated from the equation:

$n=\frac{\delta_{S}}{t}+n_{L}$ 
Where $n_{L}=1.484$ the refractive index of liquid, $t$ is the fiber thickens and $\delta_{S}$ is calculate from equation (13). We must refer that the fiber under study is isotropic therefore $n^{11}$ and $n^{1}$, so that the difference between $n^{11}$ and $n^{1}$ is zero. Figure (4) shows the spectral dispersion curve of multi-mode step index optical fiber [Ge-B doped $\mathrm{SiO}_{2}, \mathrm{r}_{\text {core }}=10.8 \mu \mathrm{m}$ Diameter $24 \mu \mathrm{m}$ ], which is obtained from the manual (VAWI). In this technique there is a limitation on fiber diameter. If the fiber diameter more than fifty $\mu \mathrm{m}$, the duplicated fringe shifts will overlapped. The accuracy of this technique depends on the accuracy of the Pluta microscope for the refractive index $( \pm 0.001)$ and the accuracy of calibration curve $b(\lambda)$. The dispersion curve of the optical fiber enables us to detect a concept of mechanism of energy losses in optical fiber, as a function of material dispersion, will be our future work.

\section{Conclusion}

Two-beam interference (VAWI) technique is used to determine the refractive index of a step index optical fiber sample. This technique uses Pluta polarizing interference microscope modified to determine the refractive index of core-cladding fiber. The (VAWI) method is a simple in operation and requires no special preparation of the samples, it enables a relatively fast measurement of the optical path differences within the whole visible spectrum, which is especially important for the measurements of the spectral dispersion of the refractive index. The method allows us to identify the interference orders, in case when the shift inside the fiber core is too large and one can not detect precisely the interference order. 


\section{ACKNOWLEDGEMENT:}

The authors would like to express their greatest gratitude to Prof. Dr. Ahmed Amin Hamza, Professor of Physics, and President of Mansoura University for useful discussions, guidance and helpful advice to complete this work. Thanks also to Prof. Dr. Taha Zakky Nabawy Sokkar, Professor of Physics; Faculty of Science, Mansoura University, and Dr. Mohamed Mabrouk, Assoc. Pof. of Physics, Faculty of Science, Mansoura University, Demietta, for their valuable support.

\section{REFERENCES:}

[1] Crisp, J., Introduction to fiber optics, Newnes, London, (2000).

[2] Pluta, M. J. Microscopy, 96, 309, (1972).

[3] Hamza A.A. and Sikorski J., J.Microsc.,113, 15,(1978)

[4] Hamza A.A., Textile Res. J., 50, 731 (1980).

[5] Źurek, W. and Zakrzewski, S., J.Appl. polym. Sci., 28, 277, (1983).

[6] Hamza A.A., J. of Microscopy, 142, 35. (1986)

[7] Hamza A.A. and Abd El-Kader. H., Phys.Ed., 21, 244, (1986).

[8] Hamza A.A. and EL- Farahaty, K A., TextileRes. J., 56, 580 (1986)

[9] Hamza A.A. and EL-Dessouki, T., Textile Res. J., 57, 508 (1987)

[10] Hamza A.A., Ghander, A.M., Sokkar, T.Z.N., Mabrouk, M.A., and Ramadan, W. A., Pure and Appl.Optics, 3, 943 (1994).

[11] Wemple, S. H., Appl. Optics, 18, 31, (1979)

[12] Mynbaev, D. K., and Scheiner, L., "Fiber-Optic Communications Technology", Hall, Inc., London (2001).

[13] Pluta, M., J. Microsc., 145, 191, (1987).

[14] Pluta, M., J. of Microscpy, 149, 97, (1988).

[15] Pluta, M., Opt.Applicata, 15, 375, (1985).

[16] Pluta, M., Opt.Applicata, 20,259, (1990). 

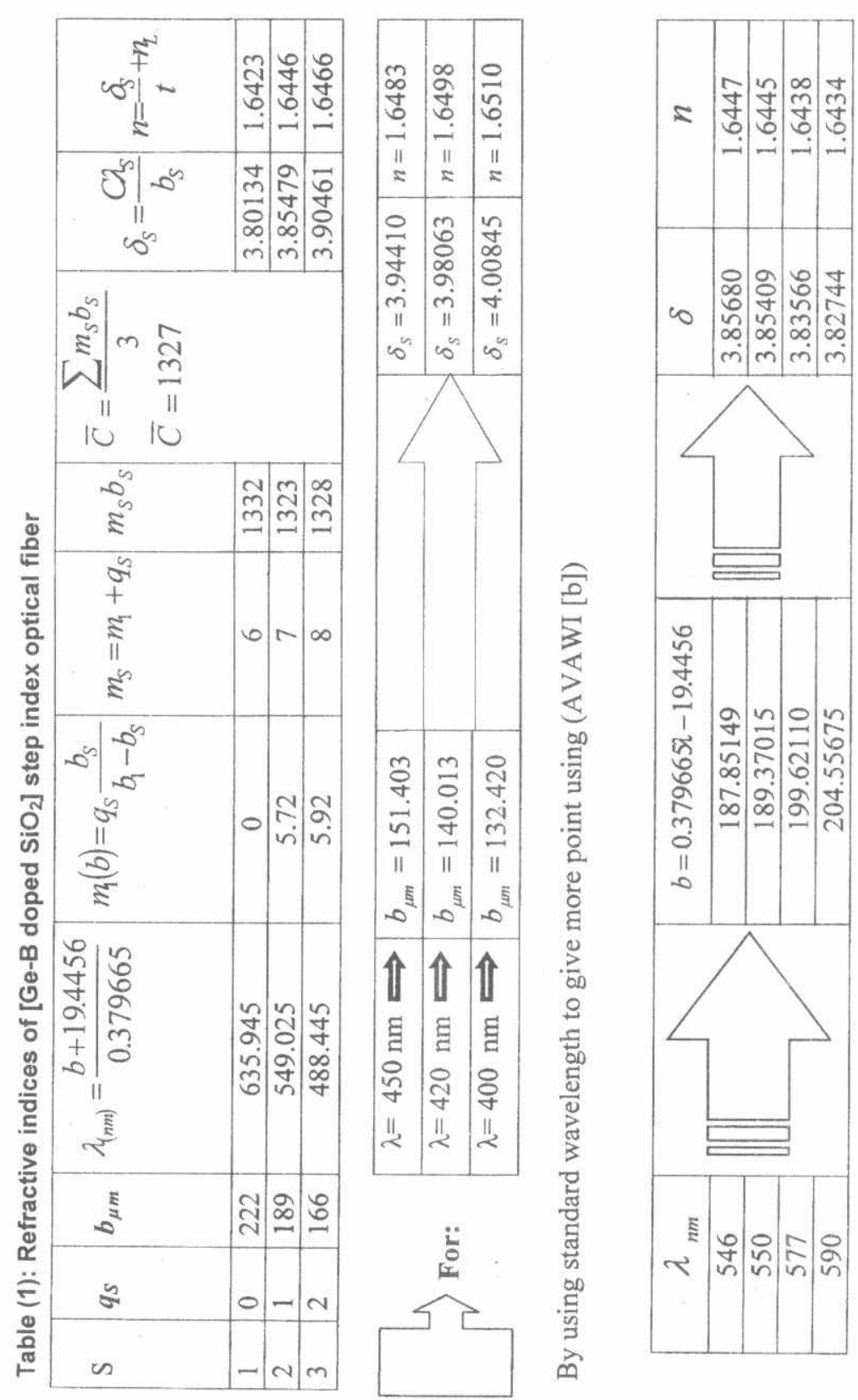

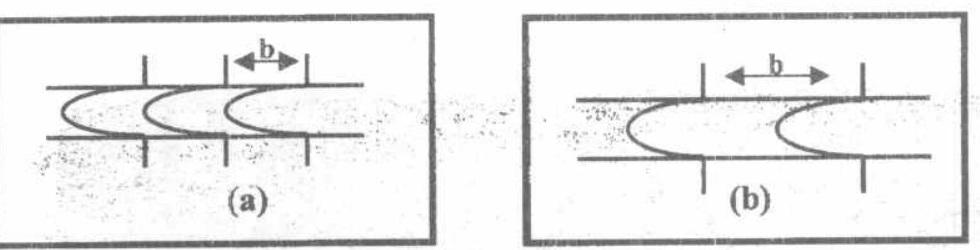

Fig.1. The coincidence (a) and anti-coincidence (b) of the fiber fringe shift

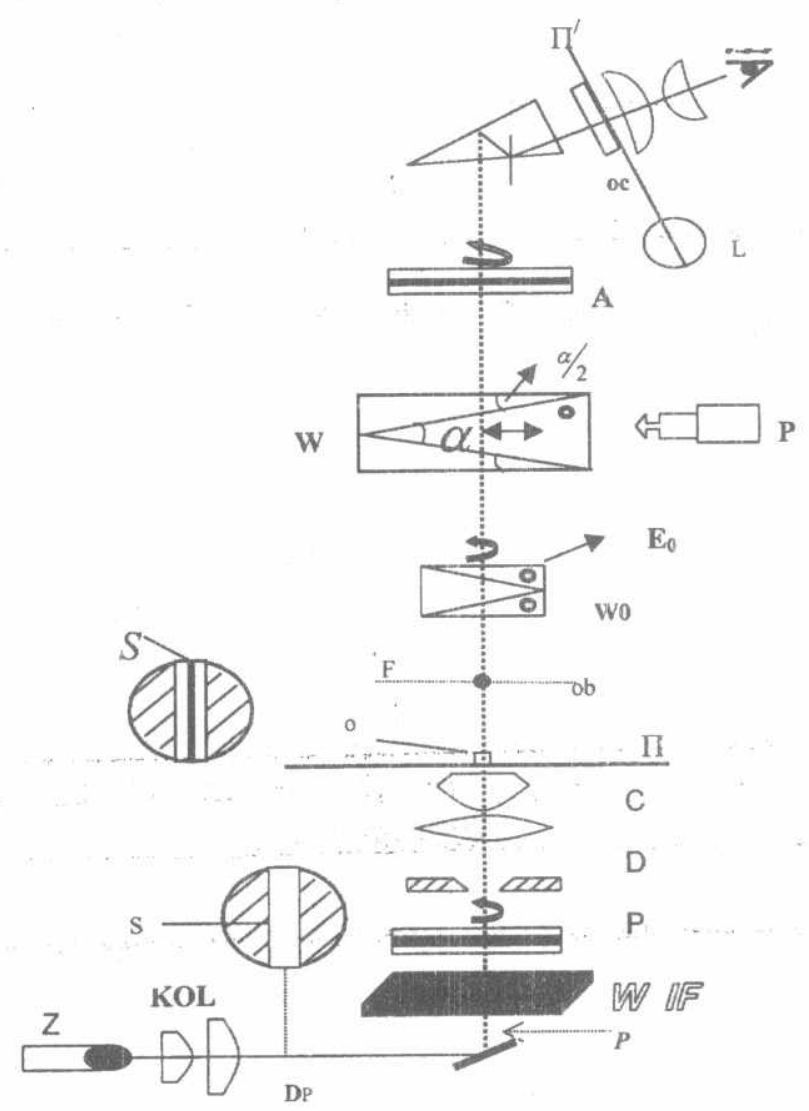

Fig.2. Optical system of double refracting microinterferometer (Pluta modified) $W_{0}$ main double-refracting prism, $W_{2}$ Wollaston prism, $C$ condenser, $D$ slit diaphragm, $Z$ halogen source, $P$ polarizer, $A$ analyzer, IF interference [16]. 


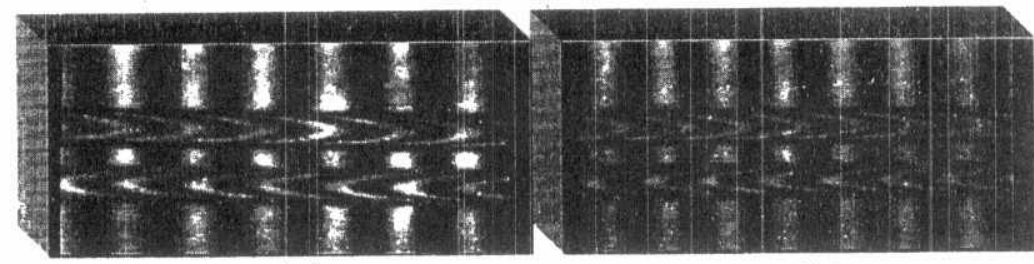

(a)

(b)

Fig .3. The coincident (a) and anti-coincident (b) position of fringes

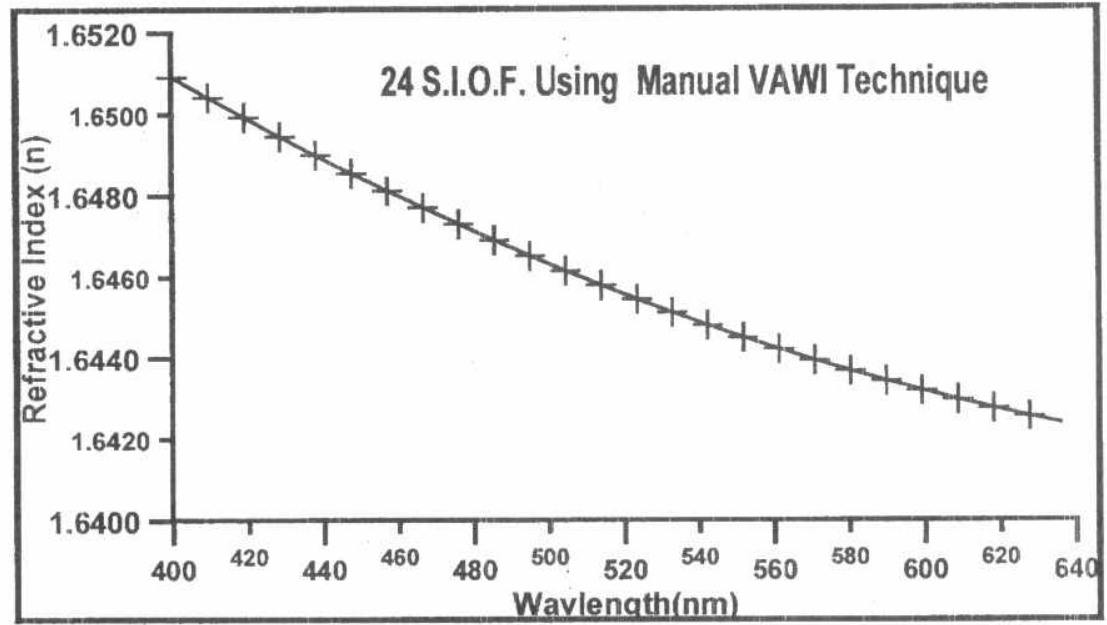

Fig.4. The spectral dispersion curve obtained using the manual (VAWI). 